\title{
Evaluation of the geometry of ionospheric current systems related to rapid geomagnetic variations
}

\author{
S. V. Apatenkov ${ }^{1}$, V. A. Sergeev ${ }^{1}$, R. Pirjola $^{2}$, and A. Viljanen ${ }^{2}$ \\ ${ }^{1}$ Institute of Physics, St. Petersburg State University, Ulyanovskaya 1, Petrodvoretz, 198504, St.Petersburg, Russia \\ ${ }^{2}$ Finnish Meteorological Institute, Geophysical Research Division, P.O. Box 503, FIN-00101 Helsinki, Finland
}

Received: 13 September 2002 - Revised: 14 March 2003 - Accepted: 12 May 2003 - Published: 1 January 2004

\begin{abstract}
To learn about the geometry and sources of the ionospheric current systems which generate strong geomagnetically induced currents, we categorize differential equivalent current systems (DEC) for events with strong $d B / d t$ by decomposing them into the contributions of electrojet-type and vortex-type elementary systems. By solving the inverse problem we obtain amplitudes and locations of these elementary current systems. One-minute differences of the geomagnetic field values at the IMAGE magnetometer network in 1996-2000 are analysed to study the spatial distributions of large $d B / d t$ events. The relative contributions of the two components are evaluated. In particular, we found that the majority of the strongest $d B / d t$ events (100-1000 nT/min) appear to be produced by the vortex-type current structures and most of them occur in the morning LT hours, probably caused by the Ps6 pulsation events associated with auroral omega structures. For strong $d B / d t$ events the solar wind parameters are shifted toward strong (tens nT) southward IMF, enhanced velocity and dynamic pressure, in order for the main phase of the magnetic storms to occur. Although these events appear mostly during magnetic storms when the auroral oval greatly expands, the area of large $d B / d t$ stays in the middle part of the auroral zone; therefore, it is connected to the processes taking part in the middle of the magnetosphere rather than in its innermost region populated by the ring current.
\end{abstract}

Key words. Geomagnetism and paleomagnetism (rapid time variations) - Ionosphere (auroral ionosphere; ionospheric disturbances)

\section{Introduction}

Geomagnetically induced currents (GIC) in long conductor systems, like power transmission systems or pipelines, are caused by rapid changes in the Earth's magnetic field (e.g.

Correspondence to: S. Apatenkov

(apatenkov@geo.phys.spbu.ru)
Boteler et al., 1998; Viljanen and Pirjola, 1994). GICs are driven by the horizontal electric field induced at the Earth's surface due to time-varying or fast-moving strongly inhomogeneous ionospheric current systems and affected by the Earth's conductivity structure. Close correlation between GIC and the time derivative of the magnetic field $(d B / d t)$ has been demonstrated by Viljanen (1998) and Viljanen et al. (2001), so in the present paper we base our analysis of potential GIC candidate events on one-minute magnetic field differences. A large variety of ionospheric current systems exist which differ by their time and spatial scales and motions. Examples of the most intense systems are the eastward and westward auroral electrojets (AEJ), westward travelling surges (WTS), and auroral omega bands (Untiedt and Baumjohann, 1993). The two latter systems have very complicated structures, including a pronounced vortex part. Here and thereafter we discuss the equivalent current systems (ECS), closed completely in the ionosphere, since the real 3-D system of ionospheric and field-aligned currents cannot be uniquely determined by using only ground magnetic field measurements, (Fukushima, 1976), as its curl-free part does not produce any disturbance on the ground.

Until recently, most of the GIC studies concentrated on considering effects of the simplest one-dimensional current system, an east-west aligned auroral electrojet (Albertson and Van Baelen (1970); Boteler et al. (1997); Towle et al. (1992)). However, a recent survey of $d B / d t$ occurrence and their directional distribution (Viljanen, 1997; Viljanen et al., 2001) indicated that many large GIC events display a large contribution from the east-west $d B / d t$ component, especially in the morning LT hours at auroral latitudes, which cannot be explained by AEJ effects. The detailed study of the April 2000 storm by Pulkkinen et al. (2003) also demonstrated the complexity of ionospheric currents during an extreme GIC event.

Some attempts have been done to consider more complicated geometries of ECS, for example, in model studies of a real power system in works by Viljanen et al. (1999) and Pulkkinen et al. (2000). They concluded that large GIC could 
be most probably produced by rapid intensification of AEJ or fast moving WTS. They also discussed the origins of GIC events and found about half of the events to be caused by electrojet variations. However, the rough characterization was based only on the magnetogram shape and is thus not very reliable.

In this paper we address the question of how often is the simple AEJ model adequate for GIC modeling, and, specifically, how large can the contribution from non-onedimensional systems can be during large $d B / d t$ events. To characterize it in a quantitative way, we represent the equivalent currents as a sum of the simplest 1-D (AEJ) and 2-D (vortex) contributions, which allows us to evaluate their relative contributions to magnetic variations observed on the dense IMAGE magnetometer network. With this simple tool we analyse the large $d B / d t$ events observed during the rising phase of the solar cycle (years 1996-2000), and study statistically the spatial distributions and solar wind conditions, paying attention to the relative complexity of the current system.

\section{Quantitative characterization of the equivalent cur- rent systems observed by the IMAGE magnetometer network}

In our approach we select the model as simple as possible, using the sum of two basic elementary current systems, a 1-D current (AEJ) and a current vortex. For the AEJ the initial model is that of the linear current aligned perpendicular to the geomagnetic meridian, where geographic components of the magnetic fields are

$$
\begin{aligned}
& B_{x}=\frac{\mu_{0} I_{0}}{2 \pi} \frac{H \cos \gamma}{H^{2}+\left(\tilde{x}-\tilde{x}_{c}\right)^{2}} \\
& B_{y}=\frac{\mu_{0} I_{0}}{2 \pi} \frac{-H \sin \gamma}{H^{2}+\left(\tilde{x}-\tilde{x}_{c}\right)^{2}} \\
& B_{z}=\frac{\mu_{0} I_{0}}{2 \pi} \frac{-\left(\tilde{x}-\tilde{x}_{c}\right)}{H^{2}+\left(\tilde{x}-\tilde{x}_{c}\right)^{2}} .
\end{aligned}
$$

Here, $\gamma$ is the angle between the geographic and geomagnetic meridians (in our case $\gamma=14^{\circ}$ ), $\tilde{x}_{c}$ is the electrojet position along the central geomagnetic meridian (referred to the point $67.5^{\circ} \mathrm{N}, 25^{\circ} \mathrm{E}$, the centre of the IMAGE network, Fig. 1); $\tilde{x}$ is the location of the observation point $\left(x_{s}, y_{s}\right)$ projected to the central geomagnetic meridian, $\tilde{x}=x_{s} \cos \gamma-y_{s} \sin \gamma$; $H$ is the height of the ionosphere assumed to be an infinitely thin layer at $110 \mathrm{~km}$ altitude above the Earth's surface, and $I_{0}$ is the current amplitude.

To reduce the number of free parameters in this model, the AEJ width is fixed, and assumed to be $100 \mathrm{~km}$. Formally, it is constructed as a sum of 10 line currents of equal amplitude displaced with a 10-km step from each other.

For the vortex we adopted one of two spherical elementary current systems introduced by Amm and Viljanen (1999), namely the divergence-free vortex current (Fig. 2). The

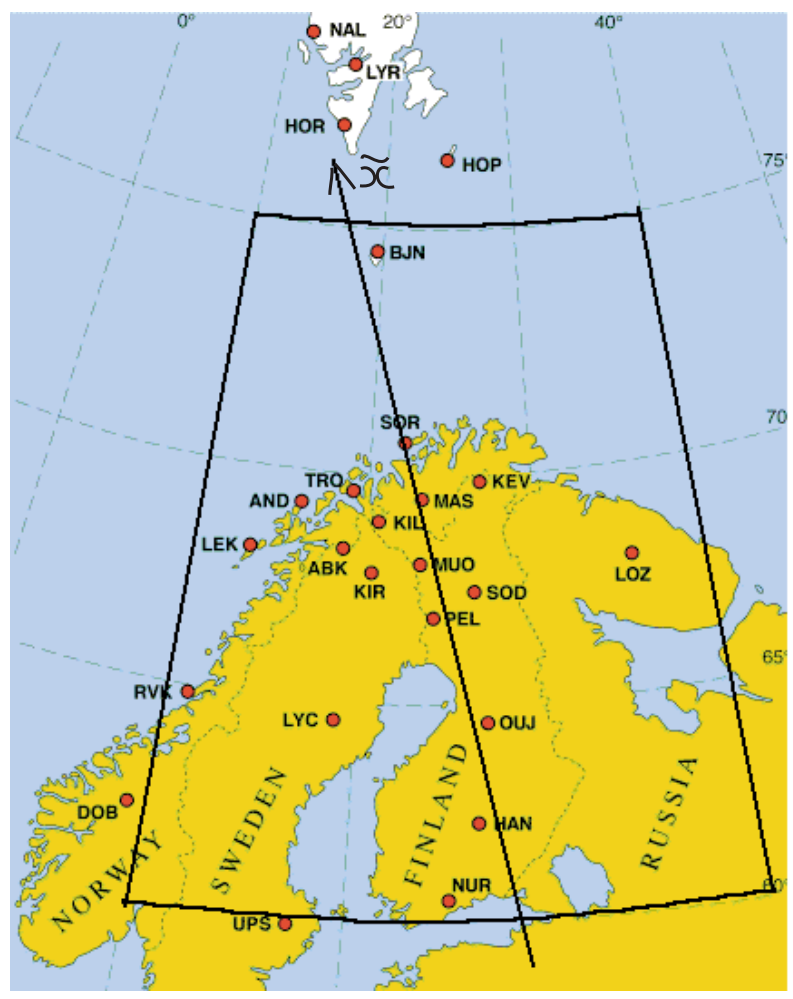

Fig. 1. IMAGE magnetometer stations in 2000. The boundaries of the grid area for the vortex locations, and the central geomagnetic meridian are also shown.

second curl-free part directly associated with a field-aligned current does not produce any magnetic effect at the Earth's surface (Fukushima, 1976) and is not of interest for us. The magnetic effect of the divergence-free system is given in spherical coordinates as

$B_{r^{\prime}}\left(r, \vartheta^{\prime}\right)=\frac{\mu_{0} I_{0}}{4 \pi r}\left(\frac{1}{\sqrt{1-\frac{2 r \cos \vartheta^{\prime}}{R_{I}}+\left(\frac{r}{R_{I}}\right)^{2}}}-1\right)$

$B_{\vartheta^{\prime}}\left(r, \vartheta^{\prime}\right)=-\frac{\mu_{0} I_{0}}{4 \pi r \sin \vartheta^{\prime}}\left(\frac{\frac{r}{R_{I}}-\cos \vartheta^{\prime}}{\sqrt{1-\frac{2 r \cos \vartheta^{\prime}}{R_{I}}+\left(\frac{r}{R_{I}}\right)^{2}}}+\cos \vartheta^{\prime}\right)$.

Here, $R_{I}$ is the ionosphere radius, i.e. $R_{I}=R_{E}+110 \mathrm{~km}$; $r$ and $\vartheta^{\prime}$ are the observer's coordinates in the reference system with $\vartheta^{\prime}=0$ at the pole of this elementary current system (see Fig. 2), and $I_{0}$ is the scaling factor.

The contribution of induced currents in the Earth are taken into account using the simple image method. Based on the studies of the Earth's conductivity structure in Scandinavia (e.g. Viljanen, 1995; Tanskanen et al., 2001), we set a perfectly conducting layer at a depth of $100 \mathrm{~km}$ for the variations with $T \sim 1 \mathrm{~min}$.

All calculations for the vortex part are carried out in a spherical frame of reference which takes into account the Earth's curvature. The AEJ part is calculated in cartesian 


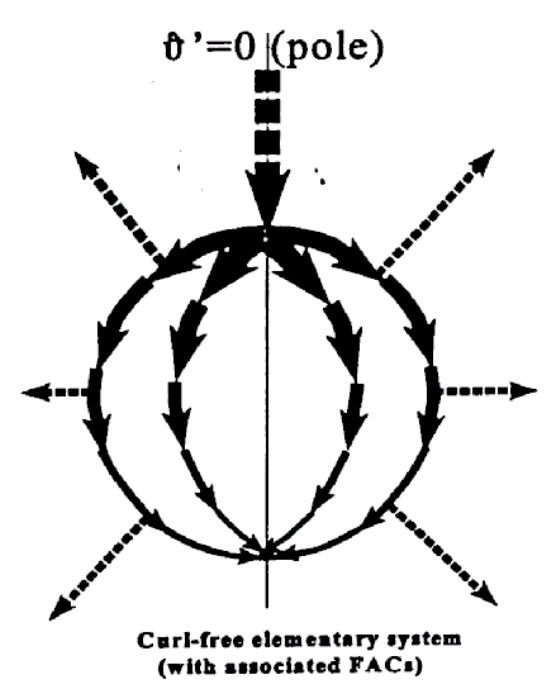

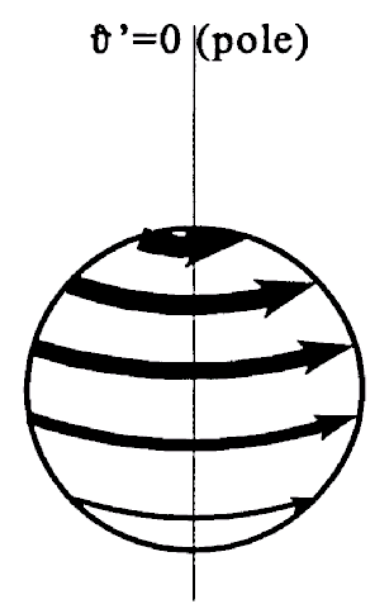

Divergence-free elementery syatem
Fig. 2. Two spherical elementary current systems used by Amm and Viljanen (1999), the curl-free part (left) and the divergence-free part (right). The divergence-free part is used in this study as a model for the current vortex. geometry for infinite long linear currents. Errors in computed magnetic field values caused by discrepancies between directions of geographic and cartesian axes, as well as by the difference between the line current and current aligned along the geomagnetic latitude at $H=110 \mathrm{~km}$ at the network edges do not exceed $10 \%$.

Our model consists of a sum of previously defined AEJ and vortex current systems, whose positions are set on the grids. The grid for the vortex (Fig. 1) covers $10^{\circ} \mathrm{E}-40^{\circ} \mathrm{E}$ in longitude and $60^{\circ} \mathrm{N}-75^{\circ} \mathrm{N}$ in latitude. The longitudinal step for possible vortex locations is $0.5^{\circ}(18-27 \mathrm{~km})$ and the latitudinal step is $0.2^{\circ}(22 \mathrm{~km})$. The latitudinal step for the AEJ model is $50 \mathrm{~km}$ and the grid covers $2000 \mathrm{~km}$ (41 possible positions) along the central geomagnetic meridian (passing through the point $67.5^{\circ} \mathrm{N}, 25^{\circ} \mathrm{E}$ ). This choice of the grid (its steps and boundaries) was decided after experiments that took into account the station spacing, the network coverage and the speed of computer calculations.

For each given set of positions $x_{\mathrm{AEJ}}$ and $x_{V}, y_{V}$ of elementary currents defined above, the intensities $I_{\mathrm{AEJ}}$ and $I_{V}$ can be determined with the standard least-square fit approach. As usually, we construct the standard deviation

$\sigma=\sqrt{\frac{\sum_{n=1}^{N} \sum_{l=1}^{3}\left(I^{V} B_{n l}^{V, m}+I^{\mathrm{AEJ}} B_{n l}^{A E J, m}-\delta B_{n l}^{o b s}\right)^{2}}{3 N}}$

and find the best fit $I_{\mathrm{AEJ}}$ and $I_{V}$ values by solving equations $\partial \sigma / \partial I_{\mathrm{AEJ}}=0$ and $\partial \sigma / \partial I_{V}=0$ for $I_{\mathrm{AEJ}}$ and $I_{V}$. Indices $n$ and $l$ in Eq. (6) stand for the station and component numbers, $N$ is the total number of available stations, $B_{n l}^{V, m}, B_{n l}^{A E J, m}$ and $\delta B_{n l}^{o b s}$ are model and observed magnetic field values at the station locations. $\delta B^{o b s}$ and $I$ are expressed in $\mathrm{nT}$ and Amperes, respectively, model fields $B^{m}$ are in nT/A, i.e. calculated for the fixed current of one Ampere.

Having the fit quality estimate $\sigma$ for the given AEJ and vortex positions, we then vary these positions on the grid to find the best fit values which have the minimal $\sigma$. Such final best fit positions and intensities of the model current system are our output parameters for each particular timestep.

A few other useful parameters are also computed. First, we can characterize the partial contributions of the AEJ and vortex as

$\sigma_{\mathrm{AEJ}}=\sqrt{\frac{\sum_{n=1}^{N} \sum_{l=1}^{3}\left(I^{\mathrm{AEJ}} B_{n l}^{A E J, m}-\delta B_{n l}^{o b s}\right)^{2}}{3 N}}$

$\sigma_{V}=\sqrt{\frac{\sum_{n=1}^{N} \sum_{l=1}^{3}\left(I^{V} B_{n l}^{V, m}-\delta B_{n l}^{o b s}\right)^{2}}{3 N}}$.

Their ratio $\left(\sigma_{V} / \sigma_{\mathrm{AEJ}}\right)$ will then characterize whose contribution is larger.

In our analysis we use as input one-minute differences of observed magnetic field values, $\delta B_{n l}^{o b s}=B_{n l}\left(t_{0}+1\right.$ min $)-$ $B_{n l}\left(t_{0}\right)$. This choice is natural, as we are interested in large $d B / d t$ events, and it also leaves aside the problem related to the choice of the absolute reference level for the variations analysed. The values $\delta B^{o b s}$ averaged over all stations give us the average variation $\left\langle\delta B>=\sum_{n=1}^{N} \sum_{l=1}^{3}\left|\delta B_{n l}\right| / 3 N\right.$. Since stronger current systems produce larger standard deviations, $\sigma, \sigma_{V}$ and $\sigma_{\mathrm{AEJ}}$ are normalized to this average disturbance magnitude $<\delta B>$ when discussing the fit quality and the partial contributions of the model current systems. To define the strong events and categorize them hereafter into different magnitudes, we use the maximal variation among disturbances of all three components at all IMAGE stations, $(\delta B)_{\max }$.

In our survey we analysed all events above the threshold $d B / d t>30 \mathrm{nT} / \mathrm{min}$. During the 5 year data interval 19962000 , covering the rising and maximal phases of the solar cycle 23, we found 39600 timesteps of such large $d B / d t$. Below we also investigate how the distributions vary with a varying $d B / d t$ threshold. Thirteen to twenty stations have 
been available during the analysed period, covering the latitudes between $60^{\circ} \mathrm{N}$ to $75^{\circ} \mathrm{N}$ (the polewardmost stations at Svalbard have not been used here). A good longitudinal coverage is available at the latitudes between $67^{\circ}$ and $72^{\circ}$, see Fig. 1.

Our approach has some limitations:

1. The simplicity of the elementary current system used poses a question of how well it represents the observed $\delta B$ distribution. This is answered by computing the ratio $\sigma /<\delta B>$. By selecting according to this parameter we can choose well-recognized events. The usage of one vortex (instead of a more complicated multiple vortex system, e.g. Amm and Viljanen, 1999) is also justified by noticing that the strongest of the known current systems have spatial scales of some hundreds to thousand km (Untiedt and Baumjohann, 1993), which is comparable to the area covered by the IMAGE network.

2. We analyse $\delta B$ - differences over 1 min which ignores the $d B / d t$ contributions from variations with periods less than $2 \mathrm{~min}$. This is justified by noticing that the time scale of the most important WTS, auroral breakup and omega bands systems is $\geq 2-10 \mathrm{~min}$. So we do not expect to miss intensifications of such current systems.

\section{Data analysis}

We start by showing the examples of the strongest $d B / d t$ events recorded on the IMAGE magnetometer network and describe the features of these events.

\subsection{Example - current systems of the strongest $d B / d t$} events

Only 10 samples with $(d B / d t)_{\max }>900 \mathrm{nT} / \mathrm{min}$ were found in 1996-2000, their observed differential equivalent currents are presented in Fig. 3. The set of events belongs to four different magnetic storms; all the events appear in the morning MLT hours (roughly MLT $=\mathrm{UT}+3 \mathrm{~h}$ for the IMAGE network). The central part of the IMAGE network is only shown since the disturbances at the southernmost stations are much smaller, in spite of the fact that all these examples appeared during very disturbed conditions $\left(K_{p}=7+\ldots 9-\right.$ and $\left.D_{s t}=-90 \ldots-240 \mathrm{nT}\right)$ when the auroral oval was greatly expanded.

Most of the patterns shown (e.g. 1, 3, 5, 8, 11) display distinct vortex structures, and the large contribution of the vortex part is also supported by a small ratio $\sigma_{V} / \sigma_{\mathrm{AEJ}}$ which was $<0.8$ for 7 out of 10 samples, see Table 1 .

The representative magnetograms from the longitudinal chain of magnetometers are shown in Fig. 4 for the storm event which gave the most of samples in Fig. 3. The tilt of the dashed lines illustrates the eastward phase motion which is also seen in samples 4, 5 and 7, 8, 9 in Fig. 3. A notable feature of the magnetograms is that the variations in the $Y$ component are comparable in magnitude with those in
Table 1. The normalised standard deviation $\sigma$ and the ratio $\sigma_{V} / \sigma_{\mathrm{AEJ}}$ for the strongest $d B / d t$ events.

\begin{tabular}{cccc}
\hline number & epoch, UT & $\sigma$ & $\sigma_{V} / \sigma_{\text {AEJ }}$ \\
\hline 1 & $04.05 .985: 32-5: 31$ & 0.80 & 0.64 \\
2 & 27.08 .98 2:21-2:20 & 0.98 & 0.97 \\
3 & $17.04 .993: 11-3: 10$ & 0.75 & 0.53 \\
4 & $22.10 .994: 04-4: 03$ & 0.73 & 0.50 \\
5 & $22.10 .994: 05-4: 04$ & 0.90 & 0.76 \\
6 & $22.10 .994: 06-4: 05$ & 1.16 & 0.70 \\
$7^{*}$ & $22.10 .994: 11-4: 10$ & 0.86 & 0.59 \\
8 & $22.10 .994: 12-4: 11$ & 0.76 & 0.53 \\
$9^{*}$ & $22.10 .994: 13-4: 12$ & 0.83 & 0.62 \\
10 & $22.10 .994: 29-4: 28$ & 1.44 & 0.82 \\
11 & $22.10 .994: 31-4: 30$ & 1.07 & 0.72 \\
12 & $22.10 .994: 56-4: 55$ & 1.13 & 1.08 \\
\hline
\end{tabular}

* samples 7 and 9 were added to show the sequence of observations

the $X$ component, as typical for vortex currents. The quasiperiodicity with $T \sim 8 \mathrm{~min}$, especially clear in the $Y$ component, is visible on the magnetograms. This pulsating behavior of the $X$ component occurs on the background of the significant negative bay.

These examples are not exotic but are well representative for a vast majority of large $d B / d t$ events. The strong contribution of the vortex part and the morning MLT occurrence will be further addressed in the following sections.

To check the relationship of these events to real GIC effects, we inspected the records of geomagnetically induced currents along the Finnish natural gas pipeline at Mäntsälä (the latitude is $\sim 60.5 \mathrm{~N}, 30 \mathrm{~km}$ east of Nurmijarvi). Data have been available since November 1998, that is for 2 of our 4 storm events shown in Fig. 3. In both cases (17 April 1999 and 22 October 1999), a significant current was recorded (4 and $7 \mathrm{~A}$, respectively), which is comparable to the maximal GIC (32 A, on 6 November and 24 November 2001) ever recorded at this station.

\subsection{Relative contributions of 1-D and 2-D current systems}

After processing the data using the algorithm described in Sect. 2 we can compare the relative contributions to the standard deviation from AEJ-type and vortex-type parts. The distributions of $\sigma_{V}, \sigma_{\mathrm{AEJ}}$ and total $\sigma$ characterizing the relative contributions and the fit quality were found to have shapes close to normal distributions, with mean values of about 1.2, 1.4 and 0.9 (in normalised units), respectively. To distinguish events with dominating AEJ- or vortex-contributions we use the parameter $\sigma_{V} / \sigma_{\mathrm{AEJ}}$ (Eqs. 7 and 8). As a threshold value to separate them we used 0.8 (based on their averages), i.e. samples with $\sigma_{V} / \sigma_{\mathrm{AEJ}}<0.8$ are considered as vortexdominated, whereas those with $\sigma_{V} / \sigma_{\mathrm{AEJ}}>0.8$ are referred to as AEJ-dominated events. Only well-recognized samples with $\sigma<1.0$ ( $\sigma$ is normalised with respect to $<\delta B>$ ) are 
(1)

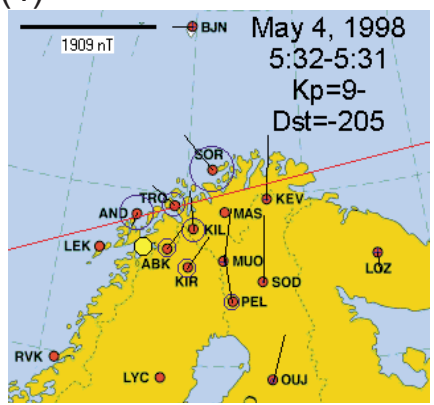

(4)

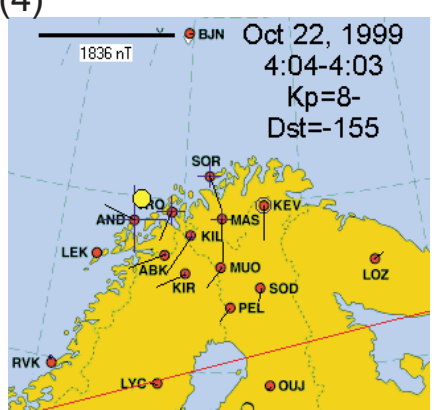

(7)

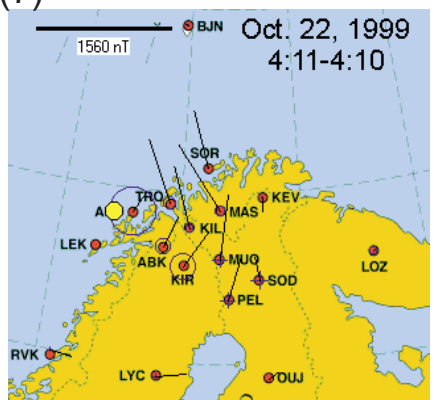

(10)

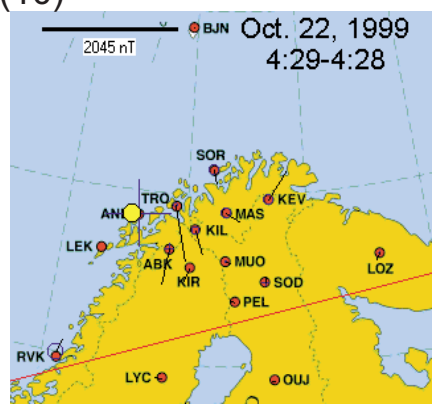

(2)

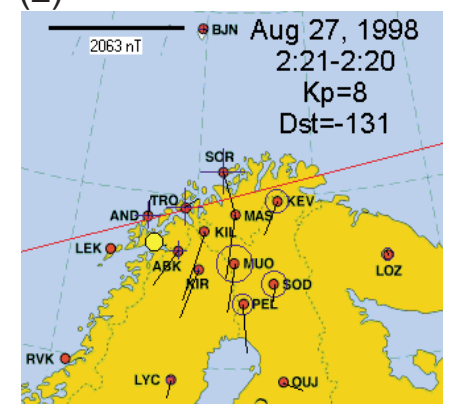

(5)

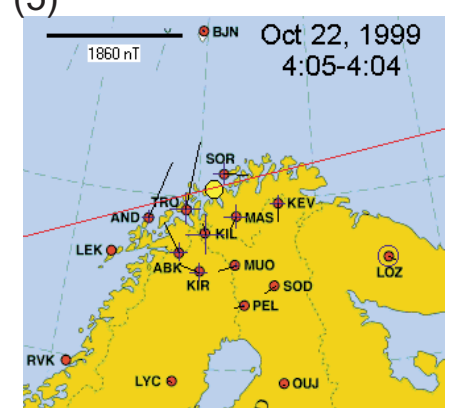

(8)

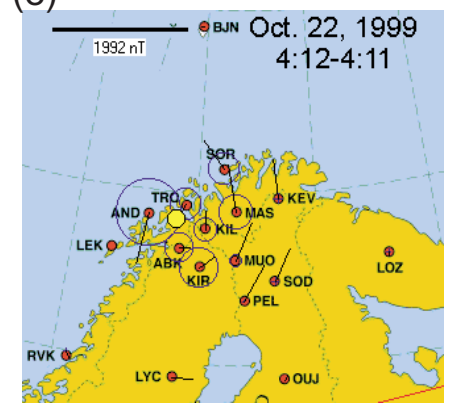

(11)

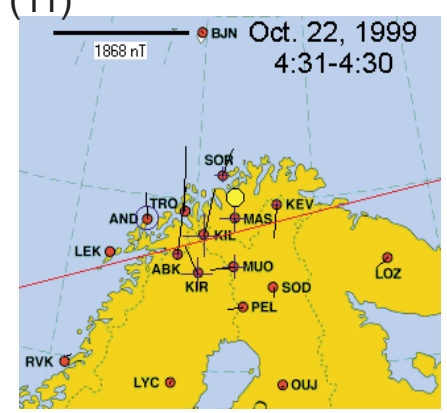

(3)

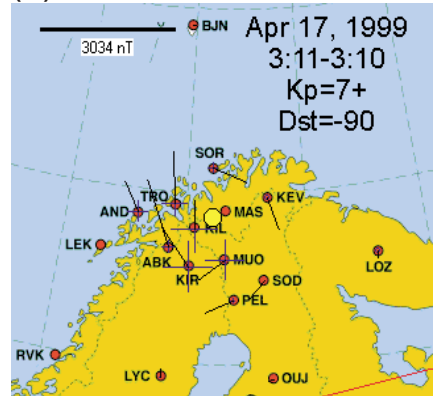

(6)

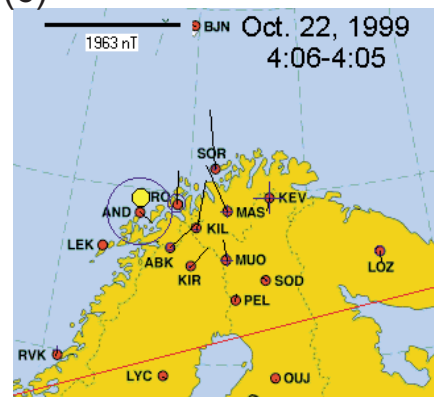

(9)

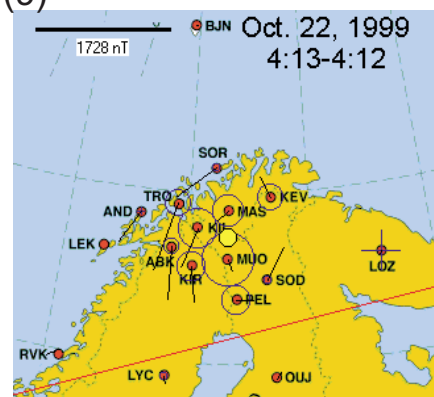

(12)

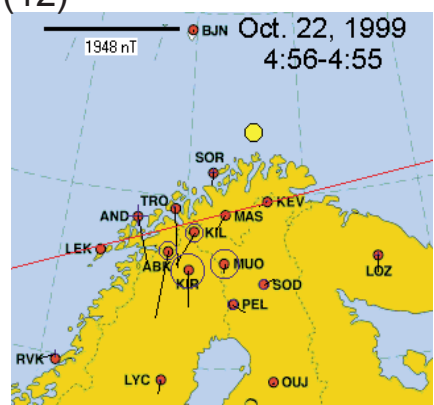

Fig. 3. Differential equivalent current patterns for ten strongest $d B / d t$ events in 1996-2000. One-minute differences of magnetic field values are shown. Arrows correspond to the horizontal magnetic field vector rotated 90 degrees clockwise, circles and crosses correspond to an upward and downward vertical component, respectively. The best fit model locations of the electrojet (red line) and the vortex (yellow circle) are also shown for comparison. Vortex-dominated pattern is distinctly seen in most cases. The samples 7 and 9 were added to illustrate the dynamics.

used in this analysis, which includes about $2 / 3$ of all events. As visual examples of the fit quality one can use Fig. 3 together with Table 1.

Figure 5 (solid line) shows two main results. First, the number of vortex-dominated and AEJ-dominated events is approximately equal even at the lowest threshold values. Second, and more importantly, the contribution of vortex- dominated samples is progressively increasing with the increase in the $(d B / d t)_{\max }$ threshold. The number of samples becomes smaller with the threshold increase, so that there are only 6 well-recognized events with $(d B / d t)_{\max }>$ $900 \mathrm{nT} / \mathrm{min}$, but the trend of the whole plot is very reliable. By choosing other limits (thresholds) for $\sigma_{V} / \sigma_{\mathrm{AEJ}}$ and $\sigma$ we obtain similar trends. This result implies that one should not 

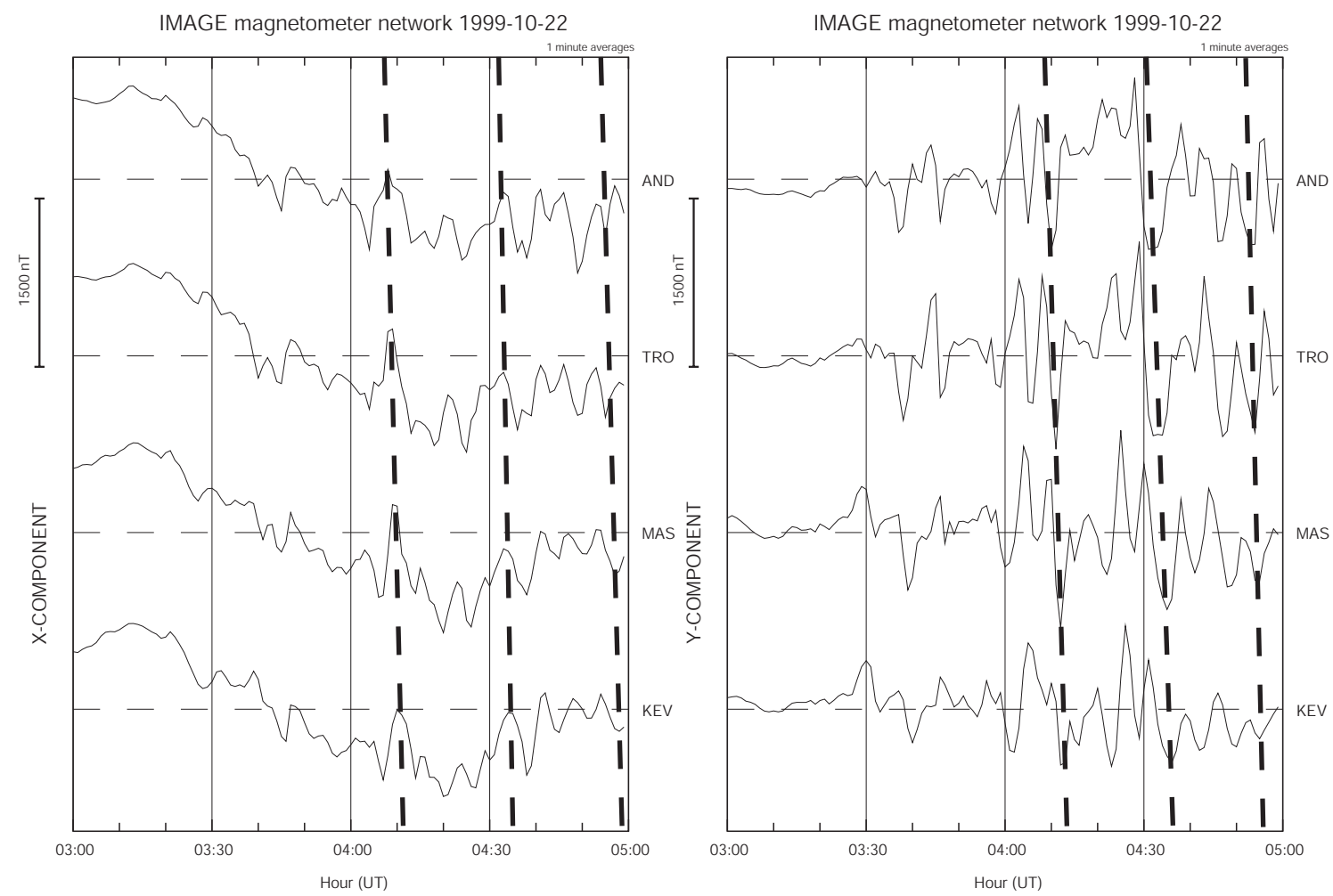

Fig. 4. Magnetograms of $X$ and $Y$ components from the longitudinal chain of IMAGE magnetometers (west to east, from AND to KEV) on 22 Oct 99 displayed in Fig. 3 (samples 4-12). Some sharp features on the magnetograms are connected with the dashed line to show the eastward phase motion. Quasi-periodic magnetic variations with $T \sim 8 \mathrm{~min}$ were observed after 04:00 UT.

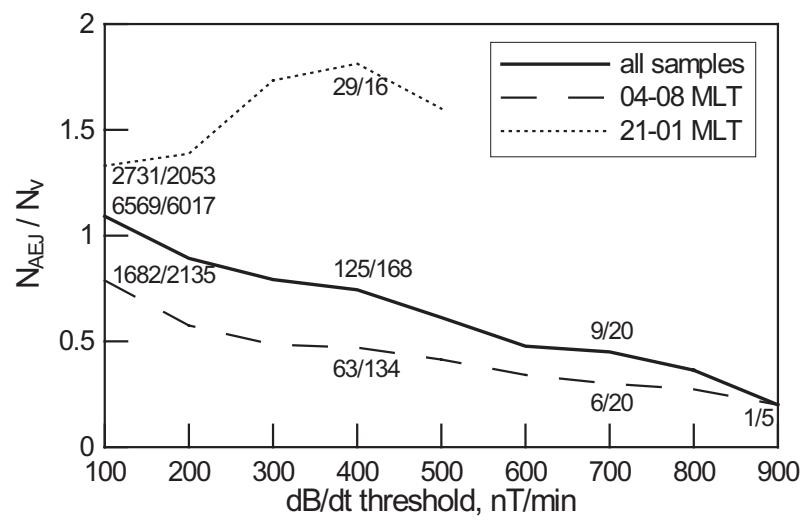

Fig. 5. Ratio of the number of electrojet-dominated samples $\left(N_{\mathrm{AEJ}}\right)$ to the number of vortex-dominated samples $\left(N_{V}\right)$ as a function of $d B / d t$ threshold. Statistics for morningside and pre-midnight samples is also shown by dashed and dotted lines, respectively, the $N_{\text {AEJ }}$ and $N_{V}$ numbers are explicitly shown for some thresholds to illustrate the statistics available.

ignore the 2-D character of the current systems when modeling the GIC effects, especially when we are interested in the largest events. Morningside and pre-midnight events are shown separately in Fig. 5 (dashed lines). The trend for the samples in the 04:00-08:00 MLT sector is the same, however, smaller amount (see subscripts along the lines in Fig. 5) of samples in 21:00-01:00 MLT have a larger electrojet contribution. As shown in the following section the number of pre-midnight samples relatively decreases for large $d B / d t$ thresholds.

\subsection{Diurnal and latitudinal variation}

Viljanen et al. (2001) showed that the occurrence of large $d B / d t$ events displays the strong MLT variation, so we start with a study of these distributions. When doing statistical analysis it is worth separating continuous series of large $d B / d t$ timesteps from isolated timesteps. (For example, a strong storm may produce a series of 1-min timesteps above some $d B / d t$ threshold, at the same time this could be one event from the GIC risk point of view.) For this purpose we repeated the occurrence analysis separately for individual 1-min timesteps (samples) and for the hours (i.e. it has at least one timestep exceeding the threshold).

Diurnal distributions for the hours with different $d B / d t$ thresholds are shown in Fig. 6. They all show two occurrence maxima in the pre-midnight and morning hours, respectively. This agrees with the results by Viljanen et al. (2001), who analysed the occurrence of events with $d H / d t>1 \mathrm{nT} / \mathrm{s}$ (60 nT/min). We, however, also see interesting differences. With the lowest threshold ( $>30 \mathrm{nT} / \mathrm{min})$ the picture resembles the pattern obtained by Viljanen et al. (2001) which has the pre-midnight maximum larger than the morning maxi- 

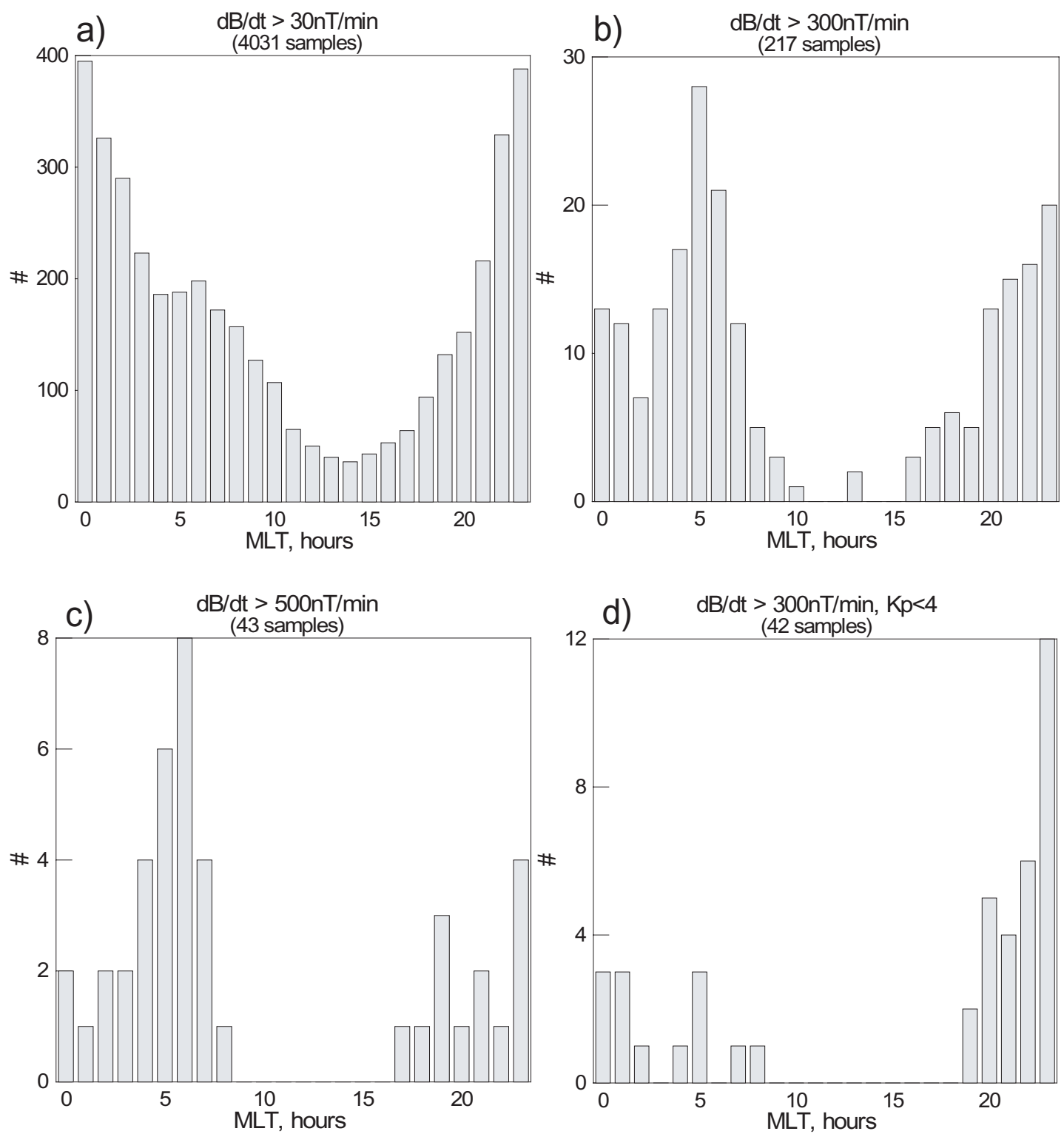

Fig. 6. MLT occurrence of hours which have at least one sample with $d B / d t$ above the given threshold. The thresholds are (a) $-30 \mathrm{nT} / \mathrm{min}$, (b) $-300 \mathrm{nT} / \mathrm{min}$, (c) $-500 \mathrm{nT} / \mathrm{min}$. (d) - the same threshold as in (b), but only small $K_{p}$ samples are included.

mum. With increasing threshold the morning maximum becomes comparable, and, then for $(d B / d t)_{\max }>500 \mathrm{nT} / \mathrm{min}$ it dominates. This is consistent with the examples of the strongest events in Fig. 3 which all occurred in the morning MLT hours.

There is an interesting exception from this trend. If we take the intermediate threshold $(d B / d t)_{\max }>300 \mathrm{nT} / \mathrm{min}$ but isolate those events which occur under a moderate disturbance level ( $K_{p}<4$, Fig. 6d), almost all morningside events disappear (although the total number of events decrease to $\sim 20 \%$ ). This is consistent with these events (occurring in pre-midnight sector) being related to moderate substorms.

The statistics obtained separately for the hours and for all timesteps give qualitatively similar results, although the numbers are different. Morning to pre-midnight maxima ratios for timesteps $(\sim 1$ and $\sim 5$ for $d B / d t$ thresholds 30 and $300 \mathrm{nT} / \mathrm{min}$, respectively) are much larger in comparison with those found for the hours $(\sim 0.5$ and $\sim 1.2$, respectively). In other words, the main difference between the distributions for the hours (Fig. 6) and those for the samples is that the morning maxima for the samples would be more pronounced. This is because the morningside events typically include the long sequences of strong variations, as seen on the magnetogram in Fig. 4. When considering the diurnal distributions separately for vortex-dominated and for electrojet-dominated samples, we obtain greater morn- 

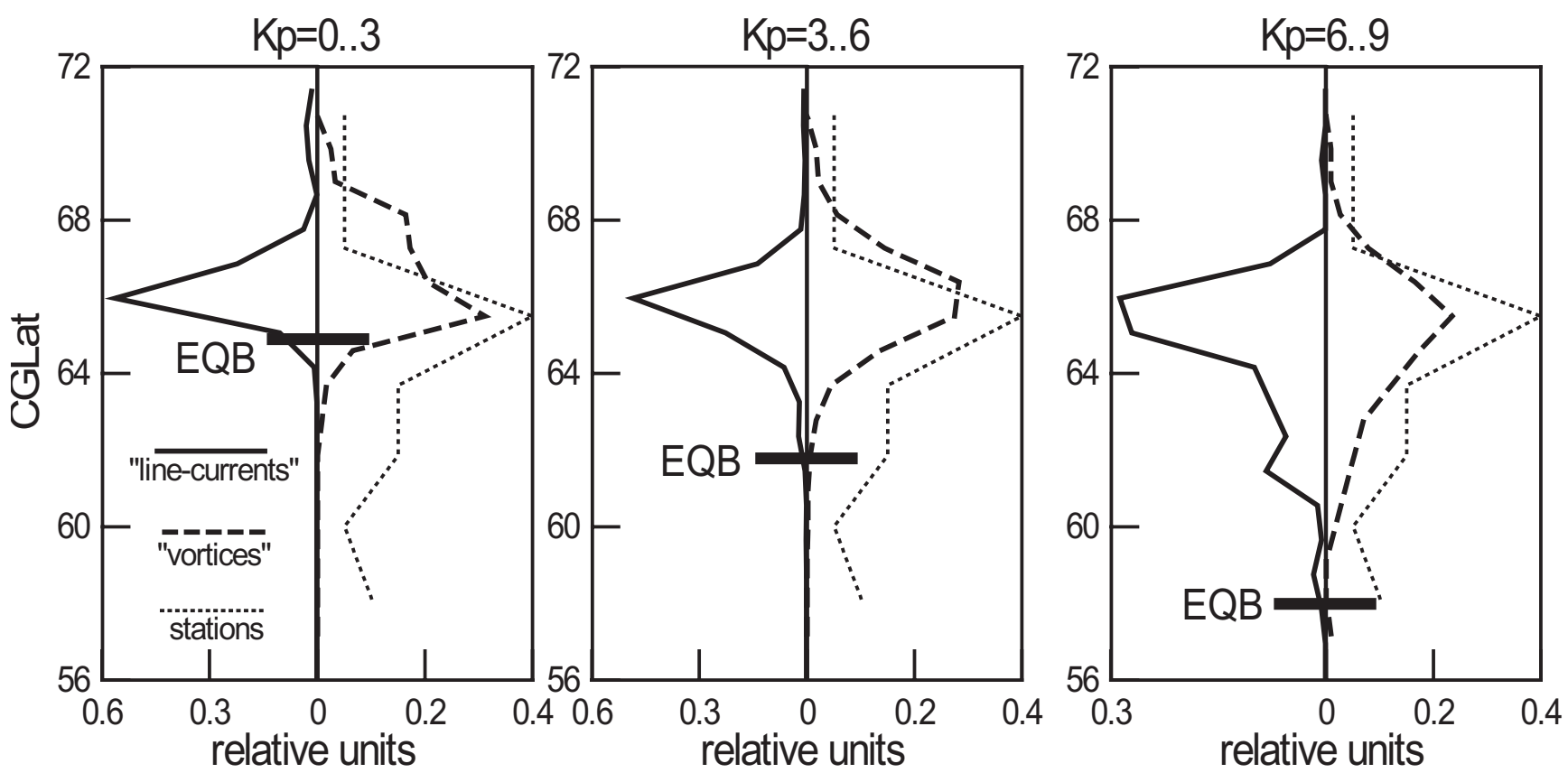

Fig. 7. Latitudinal distributions of AEJ-dominated (solid line) and vortex-dominated (dashed line) current systems. The distinction criterion as in Sect. 3.2 is applied. The latitudinal distribution of magnetometer locations is also shown for reference (dotted line). Equatorward boundary (EQB) of the auroral oval for the morning sector at MLT 4-6h is shown by horizontal ticks, its latitude near midnight would be $\sim 2^{\circ}$ equatorward (after Starkov, 1994).

ing maximum in the former case because vortex-dominated events predominantly occur in morning MLT hours (compare $N_{V}$ and $N_{\text {AEJ }}$ given in Fig. 5).

To characterize the location of the current systems in the auroral oval we also constructed the latitudinal distributions, see Fig. 7. We apply the same criterion as in Sect. 3.2 to distinguish between AEJ-dominated and vortex-dominated events. One peculiarity of Fig. 7 is that the locations of both electrojets and vortices are grouped at the latitudes which have the most dense station coverage (in the middle of network), and this changes very little with the increasing activity level. There could be two explanations of this effect. The first one is that those vortexes and jet currents which stay near the region of dense station coverage will be emphasized by the method used, since these structures would give the largest contribution to the total standard deviation which is minimized by our method. This effect is inevitably present, however, we also have strong arguments in favor of natural reasons which work in the same way, at least for the strong morningside events. The observational data plotted in Fig. 3 confirm that the largest observed $d B / d t$ are seen in the middle of observation domain, that is in the middle of the auroral zone, many degrees poleward of the expected location of the equatorward boundary of auroral oval. Evidently, the large $d B / d t$ vectors have comparable $X$ and $Y$ components, as natural for the vortex system, and these vortex structures are centered just at the latitudes where the occurrence has a peak in the Fig. 7. This has also been confirmed in the statis- tical survey by Viljanen et al. (2001), which showed that in the morning sector the large $d B / d t$ vectors have comparable $X$ and $Y$ components at these latitudes (implying that vortex structures are clustered here), whereas the polarization was more electrojet-like at the southernmost stations on the morning side, or at any latitudes in the near-midnight MLT region.

\subsection{Solar wind properties during large $d B / d t$ events}

With this large data base we can also address which solar wind conditions are efficient in producing the large $d B / d t$ events. To do so, we plot the parameter distributions in the solar wind for the events above different $(d B / d t)_{\max }$ thresholds (100, 300 and $500 \mathrm{nT} / \mathrm{min})$ and compare these distributions to those characterizing the solar wind. We used hourly averaged solar wind (OMNI) data. We tested different solar wind parameters, but show here only those which correspond to the different ways in which the solar wind influences the magnetosphere (dynamic pressure $(P d)$, which controls the magnetospheric compression, and the solar wind velocity $(V)$ and vertical IMF component $\left(B_{z}\right)$, which determine the magnetospheric electric field).

While this plot shows the expected result that strong $d B / d t$ events appear during disturbed times, the enhanced $V, P d$ and, especially, strong southward $B_{z}$ are the known conditions to result in the main phase of a magnetic storm. The plot also shows how strongly the distributions are shifted by varying the $d B / d t$ threshold. It should be noticed that the 

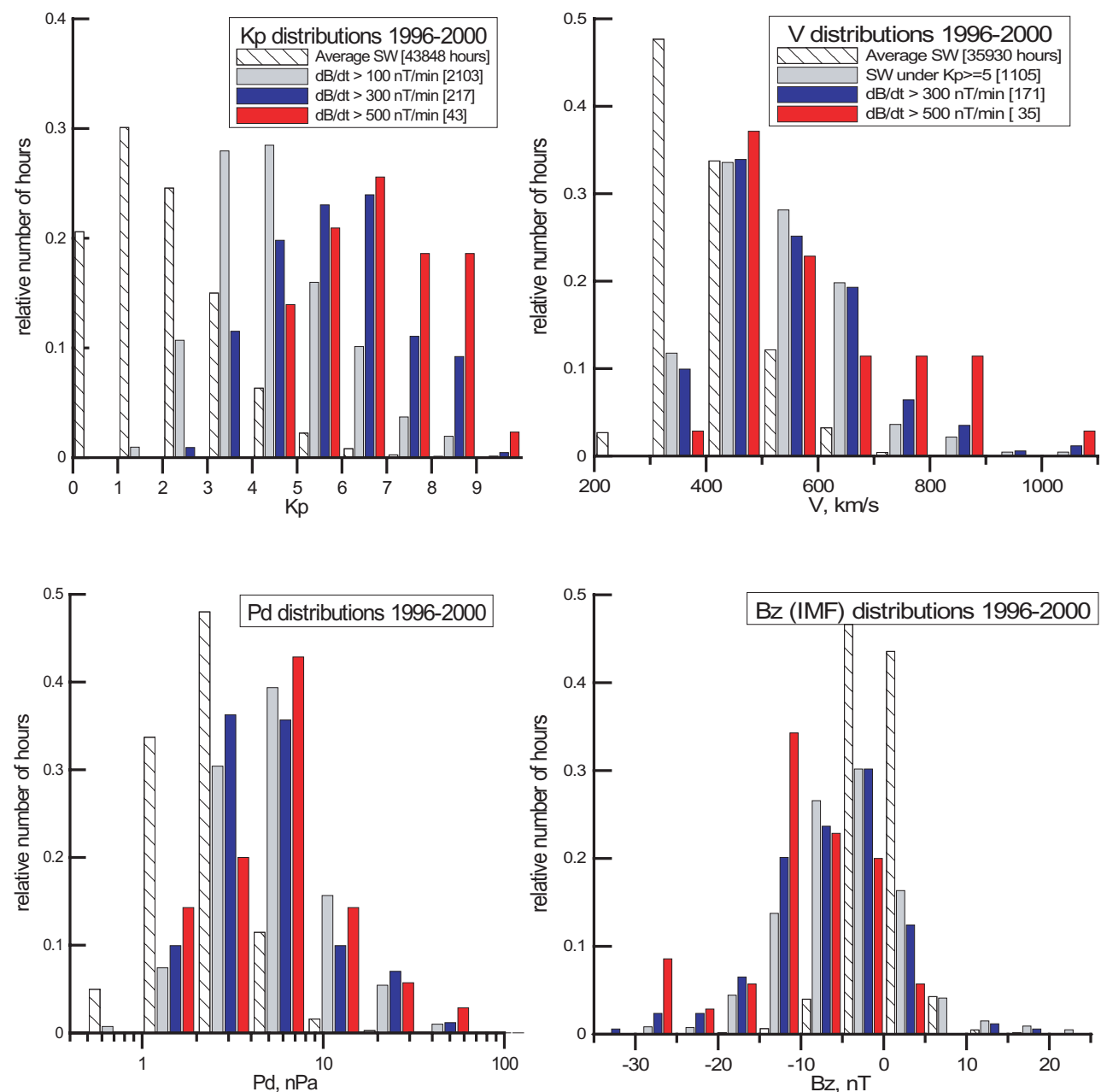

Fig. 8. Normalized occurrence distributions of hourly averaged solar wind parameters and of $K_{p}$ activity index constructed for the average solar wind as well as for large $d B / d t$ events with different thresholds. Solar wind parameter distributions for $K_{p} \geq 5$ are also shown.

strongest events with $(d B / d t)_{\max }>500 \mathrm{nT} / \mathrm{min}$ occur in the extreme tail of the solar wind parameter distributions.

We also plot $K_{p}$ distributions for different $d B / d t$ thresholds which show that the whole distribution shifts to larger $K_{p}$ with the threshold increase, and that this shift is much more pronounced than in any of the solar wind parameters (Fig. 8a). For example, events with $(d B / d t)_{\max }>300 \mathrm{nT} / \mathrm{min}$ have a maximal occurrence ratio under $K_{p} \sim 5$ and never occur under $K_{p} \leq 2$. In Fig. 8b-d we also plotted the solar wind parameter distributions for comparison. It shows that $V, P d, B_{z}$ distributions for the events under $K_{p} \geq 5$ already resemble those with the threshold $(d B / d t)_{\max }>300 \mathrm{nT} / \mathrm{min}$. This fact is quite natural, since the $K_{p}$ index is a measure of fluctuation range in the magnetic field (although at larger time scales). It means that the $K_{p}$ value is the best parameter which indicates GICs as compared to any individual solar wind parameter. However, only every fifth hour under $K_{p}>5$ includes $(d B / d t)_{\max }>300 \mathrm{nT} / \mathrm{min}$.

\section{Discussion}

Our results indicate a large contribution from vortex currents to the equivalent current systems, that produce large $d B / d t$ and, potentially, large GIC effects. Moreover, the importance of vortex currents seems to increase with an increasing $d B / d t$ threshold, so the strongest events are mostly due to the development of a transient current vortex. The obvious reason could be that the larger spatial gradients and/or violent motions are the inherent features of 2-D structures (like the auroral bulge, WTS, omega-structures, etc.) rather than of the 1-D electrojet structure. This result is in agreement with the statistics of $d B / d t$ events presented by Viljanen et al. (2001), whose Figs. 6 and 9 clearly indicate comparable contributions from $B_{x}$ and $B_{y}$ components which is an inherent property of 2-D structures as compared to the electrojetinduced magnetic field. This occurs at the stations at $64^{\circ}-67^{\circ}$ CGLat and in morning MLT hours, whereas large $B_{y}$ events were not pronounced in the pre-midnight MLT maximum (Fig. 5, dotted line). One interesting peculiarity is that dur- 
ing storm conditions, this location is in the middle of the wide and expanded auroral oval at the morningside, confirming the latitudinal distributions shown in Fig. 7. It may have important implications for the understanding of the magnetospheric sources which produce strong 2-D ionospheric current structures and, therefore, large GIC effects: they are related to the middle part of the magnetosphere rather than to the inner region occupied by the ring current.

Two peaks (in pre-midnight and morning MLT sectors) are observed in the diurnal distributions of large $d B / d t$, and they show some important differences. First, according to Viljanen et al. (2001), the polarization of the $d B / d t$ vector is mostly along the meridian in the pre-midnight events, whereas it is variable and often includes a large $Y$ component in the morning sector. This is also in agreement with our conclusion that AEJ and vortex structures are more important in the pre-midnight and the morning sectors, respectively. Second, the occurrence of pre-midnight events does not depend as much on the overall disturbance level $\left(K_{p}\right)$ as the morningside events do (our Fig. 6d). This suggests that different sources and processes could be responsible for GIC events in these two regions.

A new feature appearing in our analysis is a strong change in the $d B / d t$ event occurrence with the $d B / d t$ threshold, so that the strongest events tend to appear exclusively in the morning sector in the middle of an expanded auroral oval. A number of observed characteristics allow us to identify the source of the largest $d B / d t$ with the current system of Ps6 pulsations (e.g. Kawasaki and Rostoker, 1979) accompanying auroral omega bands and torch-like structures. These properties include: (1) morningside occurrence, (2) quasiperiodic magnetic variations with $T \sim 5-10 \mathrm{~min}$, and (3) azimuthal eastward propagation. A limited number of strong $d B / d t$ events had good coverage by Polar UVI auroral observations, and a brief inspection of these data confirm this interpretation. A more detailed investigation of auroral structures associated will be presented elsewhere.

Acknowledgements. The work on the project was supported by the INTAS project 2000-0752. S. Apatenkov and V. Sergeev thank Finnish Meteorological Institute for the support during their stays in Helsinki, their work was also supported by RFFI grant 03-0564807 and Intergeophysics Program. We thank all institutes maintaining the IMAGE magnetometer network.

The Editor in Chief thanks J. Watermann and T. Moretto for their help in evaluating this paper.

\section{References}

Albertson, V. D. and Van Baelen, J. A.: Electric and Magnetic Fields at the Earth's Surface Due to Auroral Currents, IEEE Trans. Power Appar. Syst., PAS-89, 578-584, 1970.

Amm, O., and Viljanen, A.: Ionospheric disturbance magnetic field continuation from the ground to the ionosphere using spherical elementary current systems, Earth, Planets and Space, 51, 431440, 1999.

Boteler, D. H., Boutilier, S., Bui-Van, Q., Hajagos, L., Swatek, D., Leonhard, R., Hughes, B., Ferguson, I. J., and Odwar, H. D.: Geomagnetic Hazard Assessment, Phase 2, Final Report, CEA project 357 T 848A, GSC Open File 3140, 1997.

Boteler, D. H., Pirjola, R. J., and Nevanlinna, H.: The effects of geomagnetic disturbances on electrical systems at the earth's surface, Adv. Space Res., 22, 17-27, 1998.

Fukushima, N.: Generalized theorem of no ground magnetic effect of vertical currents connected with Pedersen currents in the uniform conducting ionosphere, Rep. Ionos. Space Res. Japan., 30, 35-40, 1976.

Kawasaki, K. and Rostoker, G.: Perturbation magnetic fields and current systems associated with eastward drifting auroral structures, J. Geophys. Res., 84, 1464-1480, 1979.

Pulkkinen, A., Viljanen, A., Pirjola, R., and BEAR Working Group: Large geomagnetically induced currents in the Finnish highvoltage power system, Finnish Meteorological Institute, Reports, 2000:2, 99 pp., 2000.

Pulkkinen, A., Thomson, A., Clarke, E., and McKay, A.: April 2000 geomagnetic storm: ionospheric drivers of large geomagnetically induced currents, Ann. Geophysicae, 21, 709-717, 2003.

Starkov G. V.: A Mathematical Description of Auroral Luminousity Boundaries, Geomagn. Aeron., 34, 3, 80-86, 1994.

Tanskanen, E. I., Viljanen, A., Pulkkinen, T. I., Pirjola, R., HÄkkinen, L., Pulkkinen, A., and Amm, O.: At substorm onset, $40 \%$ of $A L$ comes from underground, J. Geophys. Res., 106, 13 119-13 134, 2001.

Towle, J. N., Prabhakara, F. S., and Ponder, J. Z.: Geomagnetic effects modelling for the PJM interconnection system. Part I Earthsurface potential computaion, IEEE Trans. Power Syst., 7, 949-955, 1992.

Untiedt, J. and Baumjohann, W.: Studies of polar current systems using IMS Scandinavian Magnetometer Array, Space Sci. Rev., 63, 245-390, 1993.

Viljanen, A., Kauristie, K., and Pajunpää, K.: On induction effects at EISCAT and IMAGE magnetometer stations, Geophys. J. Int., 121, 893-906, 1995.

Viljanen, A.: The relation between geomagnetic variations and their time derivatives and estimation of induction risks, Geophys. Res. Lett., 24, 6, 631-634, 1997.

Viljanen, A.: Relation of Geomagnetically Induced Currents and Local Geomagnetic Variations, IEEE Trans. Power Delivery, 4, 1285-1290, 1998.

Viljanen, A., Amm, O., and Pirjola, R.: Modeling Geomagnetically Induced Currents During Different Ionospheric Situations, J. Geophys. Res., 104, 28 059-28 072, 1999.

Viljanen, A., Nevanlinna, H., Pajunpää, K., and Pulkkinen, A.: Time derivative of the horizontal geomagnetic field as an activity indicator, Ann. Geophysicae, 19, 1107-1118, 2001.

Viljanen, A. and Pirjola, R.: Geomagnetically induced currents in the Finnish high-voltage power system - a geophysical review, Surv. Geophys., 15, 382-408, 1994. 